\title{
Tendon-derived biomimetic surface topographies induce phenotypic maintenance of tenocytes in vitro
}

$7 \quad{ }^{1}$ Institute for Complex Molecular Systems, Department of Biomedical Engineering, Eindhoven

Aysegul Dede Eren ${ }^{1}$, Aliaksei Vasilevich ${ }^{1}$, E. Deniz Eren ${ }^{2}$, Phanikrishna Sudarsanam $^{1}$, Urandelger Tuvshindorj ${ }^{1,3}$, Jan de Boer ${ }^{1}$, Jasper Foolen ${ }^{* 1}$ University of Technology, Eindhoven, The Netherlands

${ }^{2}$ Laboratory of Physical Chemistry, Department of Chemical Engineering and Chemistry, Eindhoven University of Technology, Eindhoven, The Netherlands.

$13 *$ Corresponding author

14 Email address: jfoolen@tue.nl

15 Postal address:

16 Department of Biomedical Engineering

17 Institute for Complex Molecular Systems

18 Eindhoven University of Technology

19 Building 15, Gemini-Zuid, Office 4.102 


\section{Abstract}

23 The tenocyte niche contains biochemical and biophysical signals that are needed for tendon

24 homeostasis. The tenocyte phenotype is correlated with cell shape in vivo and in vitro, and shape-

25 modifying cues are needed for tenocyte phenotypical maintenance. Indeed, cell shape changes

26 from elongated to spread when cultured on a flat surface, and rat tenocytes lose the expression of

27 phenotypical markers throughout five passages. We hypothesized that tendon gene expression

28 can be preserved by culturing cells in the native tendon shape. To this end, we reproduced the

29 tendon topographical landscape into tissue culture polystyrene, using imprinting technology. We

30 confirmed that the imprints forced the cells into a more elongated shape, which correlated with

31 the level of Scleraxis expression. When we cultured the tenocytes for seven days on flat surfaces

32 and tendon imprints, we observed a decline in tenogenic marker expression on flat but not on

33 imprints. This research demonstrates that native tendon topography is an important factor

34 contributing to the tenocyte phenotype. Tendon imprints therefore provide a powerful platform to

35 explore the effect of instructive cues originating from native tendon topography on guiding cell

36 shape, phenotype and function of tendon-related cells.

37 Keywords: Soft embossing, tenocytes, dedifferentiation, redifferentiation, correlation, tendon,

38 surface topography 


\section{Introduction}

41 Tendon is a unique type of connective tissue that transmits muscle contraction forces to bones to

42 produce motion and maintain body posture [1]. In healthy tendon, a typical hierarchical

43 arrangement of parallel collagen fibrils and fibers forms a tendon unit, which in an unloaded state

44 adopts a crimp-type/wavy configuration [2]. Tendon fibroblasts, i.e. tenocytes, contribute to

45 tissue homeostasis when exposed to this highly ordered collagen extracellular matrix (ECM) by

46 producing ECM proteins and thus collagen assembly and turnover [3], [4]. However, due to its

47 low cellularity and hypovascularity, tendons possess a low regenerative capacity that results in

48 poor and slow healing [5]-[7]. Injury or tissue damage often results in an increased ratio of type

49 III collagen to type I collagen and additional deposition of glycosaminoglycans (GAG) [1]. From

50 a structural point of view, the organization of collagen fibers change from highly anisotropic to

51 more isotropic and at the micro-level, they become more angulated and the number of small-

52 diameter collagen fibers is increased [8]. At the cellular level, tenocytes become more stellate-

53 shaped [1], [8]-[10] and the expression of chondrogenic genes such as Col2al and Aggrecan

54 increases [9], [11] while expression of tendon-related markers, i.e. Tenomodulin (Tnmd) [12] and

55 Scleraxis (Scx) decreases [13].

56 Similar to in vivo, changes in tenocyte phenotype, shape and function are observed during in vitro

57 culture of tendon-derived cells on flat tissue culture plastic, which thus lacks the tendon ECM

58 niche. On flat tissue culture polystyrene, tenocytes quickly lose their elongated shape and

59 decrease the expression levels of Scx [14]. Yao et al., reported that the amount of collagen type I

60 (COL1) and decorin (DCN) protein expression levels decreased significantly from passage 0 to

61 passage 8 during in vitro culture [15]. Mazzocca et al., showed that in addition to Scx, Dcn and

62 Coll, gene expression levels of other tenocyte markers, i.e. Tenascin-C $(T n-C)$ and $T n m d$, decline 
63 from passage 0 to passage 6 [16]. This phenomenon is referred to as dedifferentiation, and is also

64 a well-known phenomenon observed in chondrocytes [17]. These results point towards the lack

65 of a healthy tendon niche, i.e. biochemical and topographical changes, driving this loss of

66 tenocyte phenotype, in analogy to changes in tissue organization in vivo that similarly affect

67 tenogenicity.

68 Various strategies were used to re-differentiate the dedifferentiated tenocytes to rescue their

69 tenocyte phenotype in vitro. Supplementing culture medium with various growth factors such as

70 VEGF [18], IGF [19] and GDF5 [20] increases expression of tenocyte marker genes including

$71 S c x$ and Tnmd. Additionally, serum-deprivation, i.e. depletion of medium-associated growth

72 factors rescued the lost tenocyte phenotype [21]. In addition, the small molecule tazaratone,

73 which targets the retinoic acid receptor, preserves tenocyte phenotype via scleraxis [22].

74 Manipulating tenocyte shape has also been used as an approach to re-differentiate

75 dedifferentiated tenocytes, and one such approach that pushes cells into an elongated shape is by

76 exposing cells to a topographical cue, i.e. a substrate composed of anisotropic fibers [23]-[32].

77 For instance, Kishore et al., used aligned collagen threads to mimic the packing density,

78 alignment and strength of native tendon, and observed an increase in gene expression of $S c x$,

79 Tnmd and Tn-C compared to the randomly organized fibers [27]. Similarly, Younesi et al.,

80 fabricated 3D-bio textiles from collagen and observed that compared to random fibers, expression

81 of Collal and Tnmd increased more than 6- and 11-fold, respectively [33]. Tu et al., promoted

82 tenogenic differentiation in vitro and tendon regeneration in vivo by aligned electrospun fibers

83 derived from tendon ECM [29]. An increase in the expression of tenogenic marker genes,

84 including Scx, Dcn and Biglycan (Bgn), was observed both in vivo and in vitro [29]. We

85 previously reported that tendon-derived cells populating a reconstituted collagen tissue, ranging

86 from uniaxially constrained with spindle-shaped cells to biaxially constrained with stellate- 
87 shaped cells, display the strongest functional remodeling capacity and tenocyte phenotype when

88 adopting a spindle-shaped morphology [34]. In another approach, we took the cell shape concept

89 one step further by using a micro-topographical screening platform with a large amount of unique

90 micro-topographies for identifying feature-characteristics associated with Scleraxis expression,

91 and revealed a strong correlation of specific cell shapes, i.e. cell and nuclear area with Scleraxis

92 levels [14]. These studies underline the impact of stimulating the tenocyte phenotype by

93 manipulating the cell shape.

94 Based on the above, we hypothesized that the topographical cues present in the tenocyte niche

95 contribute to phenotypic maintenance of tenocytes. We tested this by exploiting an imprinting

96 method to replicate the native tendon topography to polystyrene. Results of this study showed the

97 importance of the tendon topography, pushing cells in a spindle-shaped morphology, in the

98 maintenance of the tenocyte phenotype in vitro, which can shed a light on further understanding

99 of tendinopathy. 
103 Tendon-derived cells rapidly change morphology and phenotype on a flat substrate

104 Flat polystyrene surfaces were used to assess the influence of passage number on tenocyte shape

105 and tenocyte phenotype. Therefore, passage one (P1) rat tenocytes were cultured and

106 subsequently passaged four times (Fig. 1). We first investigated the changes in cell and nuclear

107 shape parameters including area, compactness, solidity and aspect ratio and changes in gene

108 expression levels of tendon-related genes. Aspect ratio is calculated by the division of the length

109 of the major axis of by the minor axis; so, the higher the value, the more elongated the cells.

110 Compactness is correlated with elongation; high compactness values indicate more elongated

111 cells. Solidity is related with cell branching and the closer its value to 1 , the more solid the cells.

112 Results showed a slight increase in cell area, i.e. cell size, with passage number (Fig. 1B). Cell

113 aspect ratio and compactness significantly decreased after P1, yet solidity remained the same in

114 all groups (Fig. 1B). The increase in the nucleus area was statistically significant between P1 and

115 P3, P4 but remained at similar values at P2 and P5 (Fig. 1B). Nucleus aspect ratio significantly

116 reduced after P2, yet compactness and solidity remained unchanged between passages (Fig. 1B,

117 supplementary file 1 containing raw data). Overall, our data indicates that with passaging of

118 tenocytes to P4 on flat tissue culture plastic, they undergo shape-related changes; cells and their

119 nucleus steadily increase in size and transform from the original spindle-shaped/elongated

120 morphology to a stellate-shaped/round morphology.

121 To further evaluate the loss in tenocyte phenotype, gene expression levels of Scx, Colla1, Col3,

122 Dcn and Bgn were measured (Fig. 1D)). Scx, which is a tenogenic transcription factor [35],

123 showed a significant decrease after passage one. A similar reduction was observed for the 
124 expression of Collal, which is the most abundant protein in the tendon extracellular matrix

125 (ECM), and Col3 [36]. Furthermore, we measured the expression of genes coding for non-

126 collagenous matrix proteins Dcn and Bgn that bind to collagen, which displayed a decreasing

127 trend from P1 to P5 [37]. With these results, we confirmed that tenocytes dedifferentiate by

128 rapidly losing their morphological characteristics and expression of tenocyte marker genes on a

129 flat surface during in vitro sub-culture.

131 The native tendon imprint as cellular niche on polystyrene

132 After establishing that with passaging, tenocytes lose their phenotype on flat polystyrene, the

133 effect of the tendon surface topography, as an isolated effect, was assessed. To this end, we used

134 the soft embossing method to imprint a native tendon topography onto polystyrene (Fig. 2). The

135 imprinting protocol involved polymerization of PDMS using a 10:1 ratio of monomers and curing

136 agent on a native tendon (Fig.2A, 3), and embossing the PMDS negative imprint on polystyrene

137 (Fig.2A,6). The imprinted material was imaged using scanning electron microscopy (SEM)

138 (Fig.2B and supplementary video 1), atomic force microscopy (Fig.2C) and profilometry (Fig.2D

139 and supplementary video 2). SEM and profilometer images reveal that native tendon can indeed

140 be imprinted onto polystyrene, and the polystyrene imprint possesses a tendon-like topography.

141 AFM analysis shows that surface topographies on tendon imprints can reach a depth of $5 \mu \mathrm{m}$.

143 Late passage tenocytes adapt early passage tenocyte morphology on tendon imprints

144 Next, the morphological response of tenocytes to the tendon imprint was assessed (Fig.3). Flat

145 control surfaces were prepared by imprinting a flat PDMS mold to the polystyrene sheets. Rat

146 tenocytes (P4) were seeded on flat and imprint surfaces and stained with phalloidin to visualize 
147 F-actin (grey) and DAPI to visualize nuclei (blue). On the flat surface, (Fig.3A\&B, top panel) a

148 spread morphology was observed, contrary to tendon imprints, where cells on each replicate

149 displayed an elongated shape (Fig.3A\&B, bottom panel).

150 We further investigated the effect of tendon topography on cell and nuclear shape parameters

151 including aspect ratio, area, solidity and compactness (Fig.3D\&E). We first visualized the

152 difference in cell shape. Rat tenocytes were exposed to either a flat surface or tendon imprint

153 topography and subsequently SEM imaging was performed (Fig.3A). On the flat surface, we

154 observed that tenocytes are more spread and stress fibers were observed (yellow arrows) (Fig.3A-

155 top panel). On tendon imprints, rat tenocytes were highly elongated (red arrow) (Fig.3A-bottom

156 panel). We observed similar results when we stained tenocytes with phalloidin to visualize the

157 actin cytoskeleton (Fig.3B) and DAPI to visualize nuclei (Fig.3C). Cell and nuclear area were

158 significantly smaller on tendon imprints compared to flat (Fig.3E\&F). Additionally, cellular and

159 nuclear aspect ratio and compactness were significantly higher on tendon imprints, compared to

160 flat (Fig.3E). Cell solidity on imprints $(0.61 \pm 0.05)$ was significantly higher and closer to 1 ,

161 compared to the flat surface $(0.51 \pm 0.04)$; indicating that cell branching was less pronounced on

162 tendon imprint. Nuclear solidity was not significantly different for both topographies. Therefore,

163 results indicate that tendon imprints induce an elongated shape and reduces cytoskeletal

164 branching, resembling the early passage tenocytes on flat polystyrene.

166 Tendon imprint topography decreases tenocyte proliferation

167 Surface topography has been identified as a regulator of cell proliferation [38] [39]. Therefore,

168 the effect of tendon imprint topography on tenocyte proliferation compared to the flat surface was

169 assessed. Rat tenocytes were thus cultured on a flat surface and tendon imprint, and after 24 
170 hours the proportion of cells in the S-phase of the cell cycle (pink-purple) to all cells (blue) was

171 measured by EdU labelling (Fig.4A\&B). On tendon imprints, 37\% of the tenocytes were EdU

172 positive whereas on a flat surface, $63 \%$ of the cells were EdU positive. This indicates that tendon

173 topography relates to a less proliferative tenocyte state.

175 Expression of SCX correlates with cellular and nuclear features

176 Tendon imprints significantly affect tenocyte cell and nucleus shape. Next, the influence of

177 tendon imprint topography on tenocyte marker gene expression was assessed (Fig.5). Therefore,

178 rat tenocytes at $\mathrm{P} 4$ were cultured on tendon imprints and flat surfaces for 24 hours and the

179 amount of SCX was measured by immunocytochemistry and subsequent quantification (Fig.5A).

180 SCX levels were significantly higher expressed on tendon imprint after 24 hours (Fig.5B), which

181 was subsequently correlated with cell shape, using a Spearmen correlation calculation. A positive

182 and significant correlation was detected of SCX level with cell compactness $\left(\mathrm{r}_{\mathrm{s}}=0.71\right)$, nucleus

183 compactness $\left(r_{s}=0.71\right)$, cell eccentricity $\left(r_{s}=0.82\right)$ and nucleus eccentricity $\left(r_{s}=0.78\right)$ (Fig.

184 5C\&D, supplementary file 2 containing raw data). In addition, a negative and significant

185 correlation was detected of SCX level with cell area $\left(r_{s}=-0.58\right)$ and nucleus area $\left(r_{s}=-0.70\right)($ Fig.

186 5C\&D). SCX level thus correlates with elongated cell and nucleus shape, driven by tendon

187 surface topography.

190 Tenogenic gene expression is maintained on tendon imprints

191 After showing that native tendon topography can increase SCX level on the short term, we

192 investigated whether tenocyte phenotype can be maintained for 7 days of culture. (Fig. 6).

193 Therefore, P4 tenocytes were cultured on flat surfaces and imprints for 7 days and gene 
194 expression levels of Scx, Collal, Sox9, Dcn, and Runx2 were measured on day 1 (D1), day 3

195 (D3) and day 7 (D7). On the flat surface, expression of tendon-related genes Scx, Dcn and Collal

196 decreased over 7 days of culturing, indicating ongoing dedifferentiation (Fig. 6A-C). Expression

197 of Sox9, a chondrogenic transcription factor that is also expressed at the tendon-bone junction,

198 also decreases on the flat surface (Fig. 6E). Lastly, Runx2 gene expression, an osteogenic-

199 associated transcription factor, significantly increased on the flat surface. Contrary, on tendon

200 imprints, gene expression levels of Scx, Dcn, Collal and Sox9 increased at D1 and subsequently

201 remained stable over the 7 days, indicating that tendon imprints can both induce a slight increase

202 in tenogenic gene expression and prevent the dedifferentiation that occurs on the flat surface (Fig.

203 6A-C, E). Lastly, gene expression of Runx2 did not increase on the tendon imprints.

204 Discussion

205 Surface topography of various organs and tissues in nature give a series of evolutionary

206 advantages and provide specialized functions. They provide cells with signals to differentiate,

207 migrate, proliferate or maintain their phenotype. The surface topography of tendon tissue is also

208 considered to provide such strong cues. However, whether the tendon topography alone, devoid

209 of other cues (e.g. ligand type and density, changing mechanical properties, etc), provides a

210 strong cellular cue remained to be explored. Therefore, the soft embossing method was exploited

211 to imprint native tendon on a polystyrene surface to study the effect of tendon surface topography

212 on cell shape, proliferation and phenotype, when compared to flat polystyrene surfaces. Results

213 of this study highlight that: 1) native tendon topography can be imprinted onto a polystyrene

214 surface to conduct tendon-related studies in vitro, 2) flat polystyrene surfaces drastically alter

215 tenogenic cell shape and phenotype, 3) cellular proliferation is decreased on imprint

216 topographies, compared to flat surfaces, 4) tenocyte phenotype is maintained on tendon imprint 
217 topographies and 5) remarkably, Scleraxis expression, a key tendon marker, beautifully correlates

218 with specific cell and nuclear shape parameters. Concordantly, results of this study imply that

219 imprinting the natural tendon surface on materials, such as polystyrene, provides a tool to control

220 tenocyte cell shape and maintenance of the tenocyte phenotype, as summarized in Fig. 7.

221 Drastic changes in cell shape of freshly isolated or early passage cells upon culturing on tissue

222 culture plastic were shown for a wide variety of cell types, including tenocytes [15], [14], [40]-

223 [45]. Vermeulen et al. and Yao et al. showed that the elongated shape of tenocytes at P0

224 transforms to stellate-shaped at P1 [15], [14], which is in agreement with the changes observed in

225 cell and nucleus shape of the current study. Similarly, our observation on the decrease in

226 tenogenic marker genes corroborate with previously published reports [14], [15], [16], indicative

227 for a dedifferentiation process. Due to the rather limited availability of the early passage cells (P0

228 or P1), dedifferentiation has become an obstacle for in vitro research and associated interpretation

229 of acquired data of many cell types. This gives rise to the interesting concept of re-differentiation

230 in order to promote/restore the native phenotype.

231 The interplay between cell shape and cell fate (e.g. differentiation, proliferation, apoptosis etc.) is

232 an established concept [55] [17]. Following that concept, because healthy tenocytes in vivo adopt

233 an elongated morphology, i.e. the nucleus and cell body, which are aligned between collagen

234 fibers, re-differentiation research has focused on pushing cells towards similar morphologies.

235 Among these approaches, cells have been exposed to anisotropic fibers [24], [27], [31], [32],

236 [46]-[52] or nano/micro-grooves [14], [26], [38], [53]. For instance, Zhu et al., reported that

237 dedifferentiated tenocytes become elongated upon culturing on microgrooves, which partially

238 reversed the dedifferentiation process [26]. A similar study by Schoenenberger et al., showed that

239 tendon fibroblasts displayed a higher expression of the tendon-related marker Mohawk (MKX),

240 COL, BGN and DCN on aligned PCL fibers, compared to random fibers [54]. Results of the 
241 current study supports the concept that enforcing a spindle-shaped tenocyte morphology is

242 beneficial for maintaining/restoring their native phenotype. Specifically, our results showed that

243 elongated cell shape, i.e. high cell aspect ratio, eccentricity and compactness, is correlated with

244 SCX levels. However, a degree of heterogeneity in cell phenotype, i.e. shape parameters and

245 SCX levels, was observed especially for tendon imprints. Explanations for this may relate to the

246 imprinting technique that potentially disturbs native architecture locally, or the fact that cells on

247 imprints are exposed to a 2D surface with more freedom to adopt a preferential shape, rather than

248 the strongly confined 3D in vivo environment. Despite that, the expression of tenocyte marker

249 genes Scx, Dcn and Collal was still shown to be stabilized on tendon imprints, contrary to a

250 decay observed on flat surfaces.

251 The observation in the current study that osteogenesis (Runx2) increased on flat surfaces,

252 compared to imprints, was also observed in another study, using osteocalcin and alkaline

253 phosphatase as osteogenic marker genes [31]. Possibly, this relates to the increased proliferation

254 rate on flat surfaces resulting in cell confluency, a phenomenon that was previously linked to

255 increased Runx2 gene expression [14], [24], [56]. Although tenocytes are the most abundant cell

256 type in tendon tissue, depending on the location of the cells within the tendon, a Sox9 positive

257 subpopulation of tenocytes exists that is located at the junction between bone and

258 tendon/ligament [57], [58]. The expression of Sox9 was previously shown to remain stable when

259 tenocytes were cultured, either cyclically stretched or left unstretched, on microgrooved

260 substrates, which induces an elongated morphology [59]. Therefore, the naturally present Sox9

261 gene expression level in elongated tenocytes, which is stabilized on imprint surfaces, is lost on

262 flat surfaces since the differentiation process is initiated. Suggestively, different topographical

263 cues present in vivo in the tendon towards the enthesis, contribute to differences in phenotype of

264 locally present cells. 
265 Healthy tendon comprises of relatively quiescent tenocytes, whereas proliferation increases

266 during the remodeling phase of tendon during healing, during which the strong tissue anisotropy

267 is absent [60]. In analogy, tendon imprints resulted in suppression of proliferation, when

268 compared to flat surfaces. Similarly, proliferation and the differentiation capacity towards the

269 tenogenic lineage of human mesenchymal stem cells is also lower on aligned collagen fibers or

270 anisotropic topographies, compared to randomly oriented substrates [61][38]. One explanation

271 for different proliferation rates on different topographies is a difference in cell shape and

272 alignment of stress fibers, as previously shown [62]. In elongated smooth muscle cells (SMCs),

273 DNA synthesis was decreased, indicating that the directed cell spreading can be positively linked

274 with DNA synthesis and thus cell proliferation [63]. A possible underlying mechanism for this

275 could relate to NOR-1, a gene involved in SMCs proliferation. NOR-1 expression is among others

276 modulated by protein kinase $\mathrm{C}(\mathrm{PKC})$. In elongated cells, the activity of protein kinase $\mathrm{C}$ was

277 shown to be decreased, which was associated with a decreased NOR-1 expression and thus

278 ultimately decreased cell proliferation [62]. Considering the similarities in shape influences

279 between SMCs and tenocytes, this could be a possible explanation. Another, or additional,

280 explanation is that changes in cell proliferation are linked to surface-topography-induced

281 mechanotransduction. Surface topography was shown to alter integrin activation and focal

282 adhesion dynamics, which affects cell proliferation [64], [65]. Flat surfaces results in specific cell

283 spreading and integrin binding, resulting in focal adhesion kinase phosphorylation and activation,

284 which in turn activates the ERK pathway, cyclin D1, and finally the $\mathrm{G}_{1}-\mathrm{S}$ cell cycle transition

285 [66]. However, activation of integrins $\alpha 5$ and $\alpha 6$ have been shown to create the opposite effect

286 [67]. This indicates that the exact mechanism by which tenocyte proliferation is coupled to cell

287 shape, surface topography, mechanotransduction pathways and specific integrin recruitment on

288 tendon imprints should further be explored. 
289 The use of tendon imprints in research comes has several advantages. Firstly, the production of

290 tendon imprints requires only PDMS, polystyrene and tools to perform soft embossing; therefore,

291 upscaling the production can be cheap and fast. Secondly, they bear the potential to be used as a

292 model system to study the molecular mechanism behind the dedifferentiation and

293 redifferentiation process, including the role of surface topographies on the activation of

294 mechanotransduction pathways. Thirdly, the imprinting technology is facile enough that it can be

295 applied to any polymer that does not degrade above $140^{\circ} \mathrm{C}$. Therefore, in addition to the influence

296 of healthy tendon surface topography, the effect of other polymers on tenocyte phenotype can be

297 investigated. Finally, the technique also allows imprinting the pathogenic or damaged tendon

298 topography on polystyrene; therefore, the role of altered surface topography on tenocyte

299 phenotype can be further explored in vitro.

300 Conclusion

301 This study shows that tenocyte cell shape and expression of tenogenic marker genes drastically

302 change during in vitro subculture, which leads to decreased tenogenic characteristics.

303 Furthermore, we demonstrated that tendon imprints, which carry the topological cues from the

304 native tendon, led to an elongated cell shape and resulted maintenance in the expression of

305 tenogenic-associated markers genes, which is positively correlated with elongated cell and

306 nuclear shape parameters, i.e. aspect ratio, compactness and eccentricity. Overall, results of this

307 study support the concept of cell shape-to-phenotype in tenocytes and stresses the role of surface

308 topography on tenocyte phenotype.

309

\section{Data availability statement}

310 All data is available upon request. 


\section{Acknowledgements}

312 This research has received funding from the European Union's Horizon 2020 research and

313 innovation programme under the Marie Sklodowska-Curie grant agreement No 676338.

\section{Disclosure statement}

315 Authors do not have any financial interests to declare.

\section{Appendix A. Supplementary data}

\section{Materials and Method}

\section{Tendon tissue imprinting}

319 Fresh porcine Achilles tendons were obtained from crossbreeds of Great Yorkshire and Dutch

320 land pigs aged between 6-8 months old and between 85-95 $\mathrm{kg}$ of weight, supplied by a local

321 slaughterhouse (Compaxo Meat B.V, the Netherlands). Muscle, fat, bone-like tissues, synovial

322 sheath, and paratenon were aseptically dissected and the remaining tendon was cut into $1 \mathrm{~cm}^{3}$

323 blocks and stored at $-80{ }^{\circ} \mathrm{C}$. Frozen tendons were embedded in a mixture of polyvinyl alcohol

324 and polyethene glycol (OCT, Sakura) and fixed to the cutting base plate of a cryotome (Leica

325 CM1950) after which longitudinal sections were cut with a thickness of $300 \mu \mathrm{m}$. Sections were

326 washed with PBS and stored at $-80{ }^{\circ} \mathrm{C}$ until use.

327 Tendon sections were thawed at room temperature for 30 minutes and placed in a 6-Well plate.

328 Polydimethylsiloxane (PDMS, Dow Corning Sylgard 184, 4019862) was mixed with curing

329 agent at a ratio of 10:1 (w/w) and mixed vigorously. In order to remove the air bubbles, the

330 mixture was centrifuged at $3000 \mathrm{G}$ for 10 minutes and poured on tendon sections. They were

331 allowed to cure for 48 hours at room temperature on a stable flat surface. Then, the tendon 
332 section was peeled off from the PDMS resulting in a negative imprint of a tendon on PDMS. In

333 between two glass slides, a negative imprint of PDMS was placed onto a polystyrene film

334 (PS,GoodFellow) and pressed together with clamps and incubated at $140^{\circ} \mathrm{C}$ for 30 minutes. Flat

335 surfaces were prepared by performing the same embossing method but using a flat PDMD mold.

336 In order to allow cell attachment, polystyrene surfaces were oxygen plasma treated for $45 \mathrm{~s}$ at 75

337 mTor, $50 \mathrm{sccm} \mathrm{O} 2$, and $50 \square \mathrm{W}$.

\section{Isolation of rat tenocytes}

339 Rat tenocytes were isolated from the Achilles tendon of 23 weeks old Cyp1a2ren strain rats, after

340 euthanization due their surplus status from the breeding program. Briefly, tendons were cut into

341 small pieces and digested in a buffer containing 3mg/ml collagenase type II (Worthington

342 Biochemical), 4mg/ml dispase II (Sigma-Aldrich) and 100 $\square \mathrm{U} / \mathrm{ml}$ Penicillin/Streptomycin

343 (Thermo Fisher Scientific) for 4 hours at $37^{\circ} \mathrm{C}$ in a humidified tissue culture chamber with $5 \%$

$344 \mathrm{CO}$. Then, the suspension was passed through a $70 \mathrm{~mm}$ cell strainer (Life sciences) to obtain a

345 cell-only suspension. The cell suspension was centrifuged at $300 \mathrm{G}$ for 5 minutes and re-

346 suspended in Dulbecco's modified Eagle's medium (DMEM, Sigma-Aldrich) supplemented with

$34710 \%$ fetal bovine serum (FBS), $100 \square \mathrm{U} / \mathrm{ml}$ penicillin/streptomycin. Cells were cultured in T-25

348 flasks until $70 \%$ confluency.

349 Sterilization of tendon imprints and cell culture

350 Sterilization of flat and tendon imprints was performed by incubating the materials in $70 \%$

351 ethanol for 1 hour, and remaining ethanol was air-dried under sterile conditions. Next, samples

352 were incubated with sterile PBS for 30 minutes at $37^{\circ} \mathrm{C}$ and subsequently with culture medium

353 for 30 minutes at $37^{\circ} \mathrm{C}$ before use. 
354 Rat tenocytes were seeded on the surfaces at a density of 5000 cells $/ \mathrm{cm}^{2}$ in Dulbecco's modified

355 Eagle's medium (DMEM, Sigma-Aldrich) supplemented with 10\% fetal bovine serum (FBS)

356 (Sigma-Aldrich) and 100 $\square \mathrm{U} / \mathrm{ml}$ penicillin/streptomycin (Thermo Fisher Scientific). Cells were

357 trypsinized once they reached $70 \%$ confluency. Tenocytes at passage 4 were used, unless stated

358 otherwise.

359 RNA isolation and quantitative PCR (RT-qPCR)

360 Total RNA from each sample was isolated based on the protocol described in the RNeasy Mini

361 Kit (QIAGEN). Reverse transcription was carried out based on the protocol provided by 362 iScript ${ }^{\mathrm{TM}}$ Select cDNA Synthesis Kit (Bio-Rad). Quantitative PCR was performed by using

$363 \mathrm{iQ}^{\mathrm{TM}} \mathrm{SYBR}^{\circledR}$ Green Supermix (Bio-Rad) by using the Bio-Rad CFX manager. Ribosomal Protein

364 L13a (Rpl13a) was used as a housekeeping gene and relative expression was determined using

365 the $\Delta \Delta \mathrm{Ct}$ method. Primer sequences are listed in table 1.

366 Table 1. Primer sequences used in this study

Primer Forward Primer $\quad$ Reverse Primer

name

scx GCACCTTCTGCCTCAGCAAC TTCTGTCACGGTCTTTGCTCA

colla1 ATCAGCCCAAACCCCAAGGAGA CGCAGGAAGGTCAGCTGGATAG

Col-III TGATGGGATCCAATGAGGGAGA $\quad$ GAGTCTCATGGCCTTGCGTGTTT

Den GACTCCACGACAATGAGATCACC GTTGCCATCCAGATGCAGTTC

Bgn GTTGGCCTGACGGACAGAC GCCAGCAGCAAGGTGAGTAG

Sox9 ATCTTCAAGGCGCTGCAA CGGTGGACCCTGAGATTG

Runx2 CCACAGAGCTATTAAAGTGACAGTG AACAAACTAGGTTTAGAGTCATCAAGC 
369 Cells attached to flat or tendon imprint surfaces were fixed at day 1, day 3 or day 7 after the start

370 of culturing with $4 \%$ paraformaldehyde (PFA, ThermoFisher Scientific) at room temperature for

37120 minutes and then washed with phosphate-buffered saline (PBS, Sigma-Aldrich), twice. Next,

372 samples were permeabilized with $0.5 \%(\mathrm{v} / \mathrm{v})$ Triton $\mathrm{X}-100$ in PBS for 10 minutes at room

373 temperature. After permeabilization, cells were blocked with 1:100 horse serum in PBS for one

374 hour at room temperature. Afterwards, samples were incubated in primary antibody for Scx

375 (1:200; Abcam; ab58655) dissolved in 0.01\% (v/v) Triton X-100 and 0.5\% BSA in PBS

376 overnight at $4^{\circ} \mathrm{C}$. Next, cells were washed with $0.01 \%$ (v/v) Triton X-100 and 0.5\% BSA in PBS

377 three times and incubated with anti-rabbit secondary antibody conjugated to Alexa Fluor 647

378 (1:200; ThermoFisher A27040), together with Phalloidin-Tetramethylrhodamine B

379 isothiocyanate (Phalloidin-TRITC, 1:200; ThermoFisher) in PBS with $0.01 \%$ (v/v) Triton X-100

380 and $0.5 \%$ BSA in PBS for $1 \square$ hour. Nuclei were stained with 4',6-diamidino-2-phenylindole

381 (DAPI, 1:500; Sigma-Aldrich) for 1 hour after washing. Finally, samples were mounted on glass

382 cover slides with mounting medium (Dako, Agilent). Imaging was performed by using Leica

383 DMi8 with a TIRF Multi Color microscope (Leica Microsystems CMS) with lasers at excitation

384 wavelengths of $532 \mathrm{~nm}$ and $647 \mathrm{~nm}$, for phalloidin and scleraxis respectively.

385 EdU labelling

386 In order to identify the proliferating cells, Click-iT ${ }^{\mathrm{TM}}$ EdU Cell Proliferation Kit (Invitrogen,

387 C10340) for Imaging (Thermo Fisher) was used based on the manufacturer's instructions.

388 Briefly, tenocytes were serum-starved for 24 hours prior to EdU labelling in order to set the

389 biological clock of the cells equally. Samples were fixed with $4 \%$ paraformaldehyde (PFA, 
390 ThermoFisher Scientific) at room temperature for 20 minutes and permeabilized with with $0.5 \%$

391 (v/v) Triton X-100 in PBS for 20 minutes after 24 hours of incubation in $10 \square \mu \mathrm{M}$ EdU solution.

392 Afterwards, cells were treated with EdU reaction cocktail for 30 minutes in the dark and

393 incubated in Hoechst for another 30 minutes. Images were taken with a Leica DMi8 with TIRF

394 Multi Color microscope (Leica Microsystems CMS) at 20x magnification. The reported number

395 of proliferating cells was reported as the number of EdU labelled cells/total number of cells.

\section{Atomic force microscopy (AFM) and Profilometer}

397 Tendon imprints were imaged for surface architecture by using a tapping mode atomic force 398 microscopy (AFM; XE-100, Park Systems) by using non-contact cantilevers (PPP-NCHR, Park

399 Systems). Data were recorded with XEP software and GWYDDION software was used to image

400 the data. A Keyence VK-H1XM-131 at 20x magnification was used for profilometer images.

\section{Scanning electron microscopy (SEM)}

402 Samples were fixed with $2.5 \%$ glutaraldehyde (Fisher Scientific) at room temperature for one

403 hour. Then, they were washed with distilled water 3 times for 10 minutes, dehydrated in $25 \%$,

$40450 \%, 75 \%, 90 \%$, and 100\% ethanol for 15 minutes each, and incubated in 100\% ethanol for an

405 additional 15 minutes. Next, samples were dried in Hexamethyldisilazane (HMDS) (Sigma-

406 Aldrich) for 1 hour. Prior to imaging, samples were coated with $5 \mathrm{~nm}$ gold-palladium and imaged

407 using a scanning electron microscope (SEM) (FEI Quanta 3D FEG Dual Beam).

408 Image analysis

409 An image analysis pipeline was created in CellProfiler version 3.19. To calculate expression

410 levels of SCX and other shape parameters, 3-4 random images from three biological replicates

411 was selected. Median values of all cells were per image calculated by CellProfiler and used to

412 calculate theSpearmen correlation between median SCX intensity and cell shape parameters. 


\section{Statistical analysis}

414 All statistical analyses were performed by using GraphPad Prism version 8.0 (GraphPad

415 Software, Inc., San Diego, CA). Student's t-test was performed to calculate statistical difference

416 between cell and nuclear areas. One-way analysis of variance (ANOVA) were carried out to

417 calculate the statistical difference in RT-qPCR experiments and calculate cell/nuclear shape

418 parameters between different passages and imprint vs flat surface. Spearman correlation

419 coefficient was calculated by GraphPad correlation calculation option. For all statistical analysis,

420 significance set at $\mathrm{p} \square<\square 0.05$ to determine the significance between means. All quantitative data

421 represented in this study are based on triplicated experiments.

422

423

424

425

426

427

428

429

430

431

432

433

434

435

436

437

438

439

440

\section{References}

[1] N. L. Millar, G. A. C. Murrell, and I. B. Mcinnes, "Inflammatory mechanisms in tendinopathy - towards translation," Nat. Rev. Rheumatol., vol. 13, no. 2, pp. 110-122, 2017.

[2] M. Franchi, A. Trirè, M. Quaranta, E. Orsini, and V. Ottani, "Collagen structure of tendon relates to function," ScientificWorldJournal., vol. 7, pp. 404-420, 2007.

[3] M. Benjamin, E. Kaiser, and S. Milz, "Structure-function relationships in tendons: A review," J. Anat., vol. 212, no. 3, pp. 211-228, 2008.

[4] J. G. Snedeker and J. Foolen, "Tendon injury and repair - A perspective on the basic mechanisms of tendon disease and future clinical therapy," Acta Biomater., vol. 63, pp. 18-36, 2017.

[5] S. A. Fenwick, B. L. Hazleman, and G. P. Riley, "The vasculature and its role in the damaged and healing tendon," Arthritis Res., vol. 4, no. 4, pp. 252-260, 2002.

[6] W. Fan, N. Michael, and D. Denitsa, "Tendon injuries: basic science and new repair proposals," Gen. Orthop., vol. 2, no. July, pp. 211-227, 2017.

[7] D. Docheva, S. A. Müller, M. Majewski, and C. H. Evans, "Biologics for tendon repair," Adv. Drug Deliv. Rev., vol. 84, pp. 222-239, 2015.

[8] J. Pingel et al., "3-D ultrastructure and collagen composition of healthy and overloaded human tendon: Evidence of tenocyte and matrix buckling," J. Anat., vol. 224, no. 5, pp. 
$548-555,2014$.

P. D. Clegg, S. Strassburg, and R. K. Smith, "Cell phenotypic variation in normal and damaged tendons," Int. J. Exp. Pathol., vol. 88, no. 4, pp. 227-235, 2007.

[10] S. A. Jelinsky, S. A. Rodeo, J. Li, L. V Gulotta, J. M. Archambault, and H. J. Seeherman, "Regulation of gene expression in human tendinopathy," BMC Musculoskelet Disord, vol. 12, p. 86, 2011.

[11] J. M. Archambault, S. A. Jelinsky, L. S.P., A. . Hill, D. . Glaser, and L. J. Soslowsky, "Rat Supraspinatus Tendon Expresses Cartilage Markers with Overuse," J. Orthop. Res., vol. 25, no. May, pp. 1121-1127, 2007.

[12] J. Qi et al., "Differential expression and cellular localization of novel isoforms of the tendon biomarker tenomodulin," J. Appl. Physiol., vol. 113, no. 6, pp. 861-871, 2012.

[13] N. A. Dyment et al., "The Paratenon Contributes to Scleraxis-Expressing Cells during Patellar Tendon Healing," PLoS One, vol. 8, no. 3, 2013.

[14] S. Vermeulen et al., "Identification of topographical architectures supporting the phenotype of rat tenocytes," Acta Biomater., vol. 83, 2019.

[15] L. Yao, C. S. Bestwick, L. A. Bestwick, N. Maffulli, and R. M. Aspden, "Phenotypic drift

[16] A. D. Mazzocca et al., "In vitro changes in human tenocyte cultures obtained from proximal biceps tendon: Multiple passages result in changes in routine cell markers," Knee Surgery, Sport. Traumatol. Arthrosc., vol. 20, no. 9, pp. 1666-1672, 2012.

[17] K. Von Der Mark, V. Gauss, H. Von Der Mark, and P. Müller, "Relationship between cell shape and type of collagen synthesised as chondrocytes lose their cartilage phenotype in culture [26]," Nature, vol. 267, no. 5611, pp. 531-532, 1977.

[18] A. Kraus, D. Sattler, M. Wehland, R. Luetzenberg, N. Abuagela, and M. Infanger, "Vascular endothelial growth factor enhances proliferation of human tenocytes and promotes tenogenic gene expression," Plast. Reconstr. Surg., vol. 142, no. 5, pp. 12401247, 2018.

[19] S. R. Caliari and B. A. C. Harley, "Composite growth factor supplementation strategies to enhance tenocyte bioactivity in aligned collagen-GAG scaffolds," Tissue Eng. - Part A, vol. 19, no. 9-10, pp. 1100-1112, 2013.

D. Xia et al., "GDFs promote tenogenic characteristics on human periodontal ligamentderived cells in culture at late passages," Growth Factors, vol. 31, no. 5, pp. 165-173, 2013. 
[21] M. Vijven, S. L. Wunderli, K. Ito, J. G. Snedeker, and J. Foolen, "Serum deprivation limits loss and promotes recovery of tenogenic phenotype in tendon cell culture systems," $J$. Orthop. Res., no. December 2019, 2020.

[22] S. Webb, C. Gabrelow, J. Pierce, E. Gibb, and J. Elliott, "Retinoic acid receptor signaling preserves tendon stem cell characteristics and prevents spontaneous differentiation in vitro," Stem Cell Res. Ther., pp. 1-11, 2016.

[23] K. Metavarayuth, P. Sitasuwan, X. Zhao, Y. Lin, and Q. Wang, "Influence of Surface Topographical Cues on the Differentiation of Mesenchymal Stem Cells in Vitro," ACS Biomater. Sci. Eng., vol. 2, no. 2, pp. 142-151, 2016.

[24] M. Younesi, A. Islam, V. Kishore, J. M. Anderson, and O. Akkus, "Tenogenic Induction of Human MSCs by Anisotropically Aligned Collagen Biotextiles," Adv. Funct. Mater., pp. 5762-5770, 2014.

[25] R. Peng, X. Yao, and J. Ding, "Effect of cell anisotropy on differentiation of stem cells on micropatterned surfaces through the controlled single cell adhesion," Biomaterials, vol. 32, no. 32, pp. 8048-8057, 2011.

[26] J. Zhu et al., "The regulation of phenotype of cultured tenocytes by microgrooved surface structure," Biomaterials, vol. 31, no. 27, pp. 6952-6958, 2010.

[27] V. Kishore, W. Bullock, X. Sun, W. Scott, V. Dyke, and O. Akkus, “Tenogenic differentiation of human MSCs induced by the topography of electrochemically aligned collagen threads," Biomaterials, vol. 33, no. 7, pp. 2137-2144, 2012.

[28] T. L. Popielarczyk, A. S. Nain, and J. G. Barrett, "Aligned nanofiber topography directs the tenogenic differentiation of mesenchymal stem cells," Appl. Sci., vol. 7, no. 1, 2017.

[29] T. Tu et al., "Tendon ECM modified bioactive electrospun fibers promote MSC tenogenic differentiation and tendon regeneration," Appl. Mater. Today, vol. 18, p. 100495, 2020.

[30] S. R. Caliari, D. W. Weisgerber, M. A. Ramirez, D. O. Kelkhoff, and B. A. C. Harley, "The influence of collagen-glycosaminoglycan scaffold relative density and microstructural anisotropy on tenocyte bioactivity and transcriptomic stability," J. Mech. Behav. Biomed. Mater., vol. 11, pp. 27-40, 2012.

[31] Z. Yin et al., "The regulation of tendon stem cell differentiation by the alignment of nanofibers," Biomaterials, vol. 31, no. 8, pp. 2163-2175, 2010.

[32] W. Wang et al., "Aligned nanofibers direct human dermal fibroblasts to tenogenic phenotype in vitro and enhance tendon regeneration in vivo," Nanomedicine, vol. 11, no. 9, pp. 1055-1072, 2016.

[33] M. Younesi, A. Islam, V. Kishore, J. M. Anderson, and O. Akkus, "Tenogenic Induction 
of Human MSCs by Anisotropically Aligned Collagen Biotextiles," Adv. Funct. Mater., pp. 5762-5770, 2014.

510 [34] J. Foolen, S. L. Wunderli, S. Loerakker, and J. G. Snedeker, "Tissue alignment enhances remodeling potential of tendon-derived cells - Lessons from a novel microtissue model of tendon scarring," Matrix Biol., vol. 65, pp. 14-29, 2018.

[35] R. Schweitzer et al., "Analysis of the tendon cell fate using Scleraxis, a specific marker for tendons and ligaments," Development, vol. 128, no. 19, pp. 3855-3866, 2001.

[37] Y. J.H. and H. J., "Tendon proteoglycans: Biochemistry and function,” J. Musculoskelet.

[38] W. Y. Tong et al., "Functional replication of the tendon tissue microenvironment by a bioimprinted substrate and the support of tenocytic differentiation of mesenchymal stem cells," Biomaterials, vol. 33, no. 31, pp. 7686-7698, 2012.

[39] N. R. M. Beijer et al., "Dynamic adaptation of mesenchymal stem cell physiology upon exposure to surface micropatterns," Sci. Rep., vol. 9, no. 1, pp. 1-14, 2019.

[40] G. Schulze-Tanzil et al., "Redifferentiation of dedifferentiated human chondrocytes in

[41] M. M. J. Caron et al., "Redifferentiation of dedifferentiated human articular chondrocytes: Comparison of 2D and 3D cultures," Osteoarthr. Cartil., vol. 20, no. 10, pp. 1170-1178, 2012.

[42] A. J. Mueller, S. R. Tew, O. Vasieva, and P. D. Clegg, "A systems biology approach to defining regulatory mechanisms for cartilage and tendon cell phenotypes," no. June, pp. 114, 2016.

[43] H. Holtzer, J. Abbott, J. Lash, and S. Holtzer, "the Loss of Phenotypic Traits By

[44] P. R. Tata et al., "Dedifferentiation of committed epithelial cells into stem cells in vivo," Nature, vol. 503, no. 7475, pp. 218-223, 2013.

[45] R. Pennock et al., "Human cell dedifferentiation in mesenchymal condensates through controlled autophagy," Sci. Rep., vol. 5, pp. 1-11, 2015. 
5, pp. 1527-1534, 2009.

C. Zhang et al., "Well-aligned chitosan-based ultrafine fibers committed teno-lineage differentiation of human induced pluripotent stem cells for Achilles tendon regeneration," Biomaterials, vol. 53, pp. 716-730, 2015.

[48] Z. Yin et al., "Electrospun scaffolds for multiple tissues regeneration invivo through topography dependent induction of lineage specific differentiation," Biomaterials, vol. 44, pp. 173-185, 2015.

[49] E. Chen et al., "An asymmetric chitosan scaffold for tendon tissue engineering: In vitro and in vivo evaluation with rat tendon stem/progenitor cells," Acta Biomater., vol. 73, pp. 377-387, 2018.

[50] R. D. Cardwell, L. A. Dahlgren, and A. S. Goldstein, "Electrospun fibre diameter, not alignment, affects mesenchymal stem cell differentiation into the tendon/ligament lineage," J. Tissue Eng. Regen. Med., vol. 8, no. 2014, pp. 937-945, 2012.

[51] Z. Zheng et al., "Alignment of collagen fiber in knitted silk scaffold for functional massive rotator cuff repair," Acta Biomater., vol. 51, pp. 317-329, 2017.

[52] S. K. Czaplewski, T. L. Tsai, S. E. Duenwald-Kuehl, R. Vanderby, and W. J. Li,

[53] K. Zhou et al., "Nanoscaled and microscaled parallel topography promotes tenogenic differentiation of asc and neotendon formation in vitro," Int. J. Nanomedicine, vol. 13, pp. 3867-3881, 2018.

[54] A. D. Schoenenberger, J. Foolen, P. Moor, U. Silvan, and J. G. Snedeker, "Substrate fiber alignment mediates tendon cell response to inflammatory signaling," Acta Biomater., vol. 71, pp. 306-317, 2018.

[55] C. Luxenburg and R. Zaidel-Bar, "From cell shape to cell fate via the cytoskeleton -

[56] A. English et al., "Substrate topography: A valuable in vitro tool, but a clinical red herring

[57] Y. Sugimoto et al., "Scx+/Scx9+ progenitors contribute to the establishment of the junction between cartilage and tendon/ligament," Dev., vol. 140, no. 11, pp. 2280-2288, 2012.

[58] M. R. Buckley et al., "Distributions of types I, II and III collagen by region in the human supraspinatus tendon," Connect. Tissue Res., vol. 54, no. 6, pp. 374-379, 2013. 
575

576

577

578

579

580

581

582

583

584

585

586

587

588

589

590

591

592

593

594

595

596

597

598

[59] J. Zhang and J. H. C. Wang, "Mechanobiological response of tendon stem cells: Implications of tendon homeostasis and pathogenesis of tendinopathy," J. Orthop. Res., vol. 28, no. 5, pp. 639-643, 2010.

[60] G. Nourissat, F. Berenbaum, and D. Duprez, "Tendon injury: From biology to tendon repair," Nat. Rev. Rheumatol., vol. 11, no. 4, pp. 223-233, 2015.

[61] V. Kishore, W. Bullock, X. Sun, W. S. Van Dyke, and O. Akkus, "Tenogenic differentiation of human MSCs induced by the topography of electrochemically aligned collagen threads," Biomaterials, vol. 33, no. 7, pp. 2137-2144, 2012.

[62] R. G. Thakar et al., "Cell-shape regulation of smooth muscle cell proliferation," Biophys. J., vol. 96, no. 8, pp. 3423-3432, 2009.

[63] S. J. Liliensiek, S. Campbell, P. F. Nealey, and C. J. Murphy, "The scale of substratum topographic features modulates proliferation of corneal epithelial cells and corneal fibroblast," J. Biomed. Mater. Res. Part A, vol. 79, no. 4, pp. 963-73, 2006.

[64] R. J. McMurray et al., "Nanoscale surfaces for the long-term maintenance of mesenchymal stem cell phenotype and multipotency," Nat. Mater., vol. 10, no. 8, pp. 637-644, 2011.

[65] S. Gronthos, P. J. Simmons, S. E. Graves, and P. G. Robey, "Integrin-mediated interactions between human bone marrow stromal precursor cells and the extracellular matrix," Bone, vol. 28, no. 2, pp. 174-181, 2001.

[66] R. J. McMurray, M. J. Dalby, and P. M. Tsimbouri, "Using biomaterials to study stem cell mechanotransduction, growth and differentiation," J. Tissue Eng. Regen. Med., vol. 9, no. 3, pp. 528-539, 2014.

[67] Y. Wang et al., "Integrin subunits alpha5 and alpha6 regulate cell cycle by modulating the chk1 and Rb/E2F pathways to affect breast cancer metastasis," Mol. Cancer, vol. 10, no. 1, p. 84, 2011. 

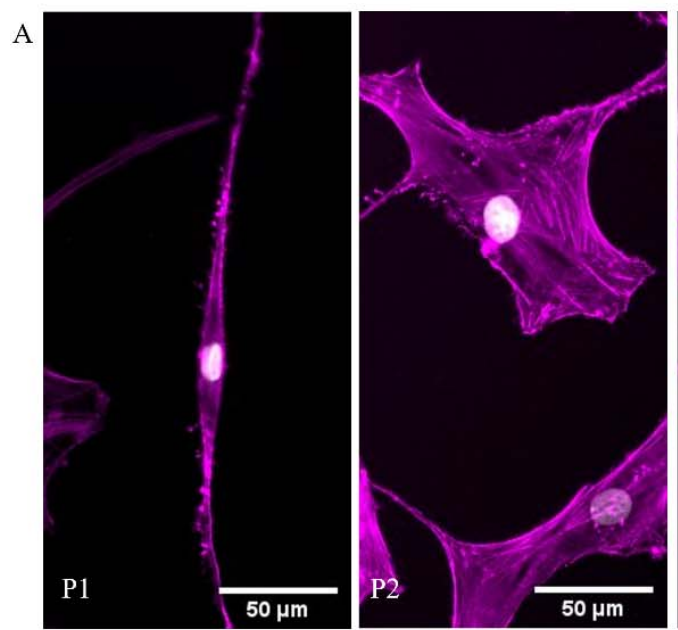

Cell area

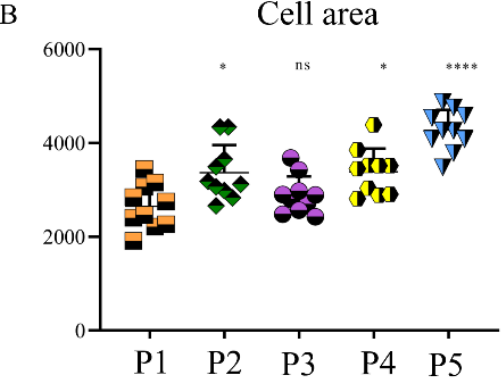

$\mathrm{C}$

Nucleus area

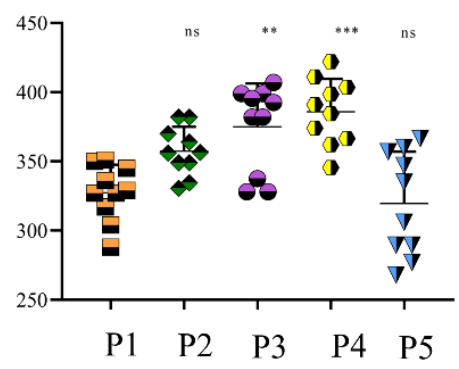

$\mathrm{D}$

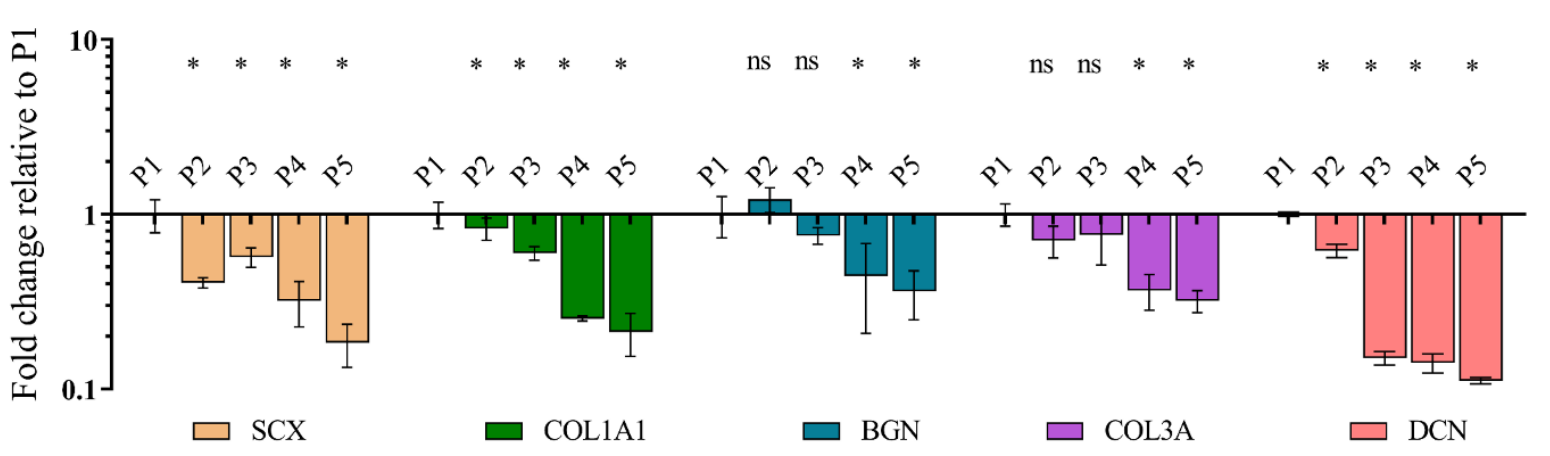

Cell shape parameters

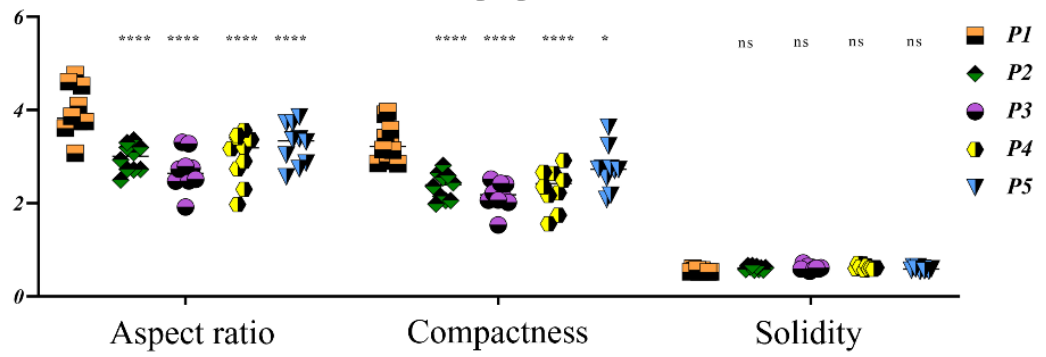

Nucleus shape parameters

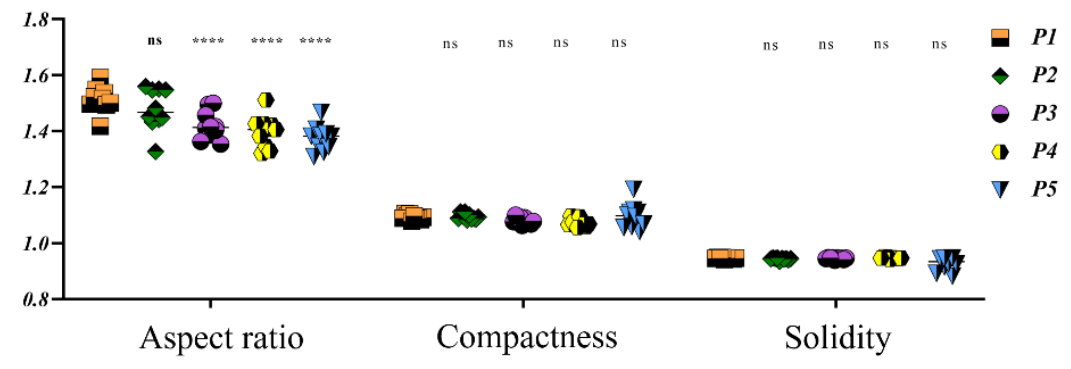

Figure 1. Tendon-derived cells rapidly lose their morphology and phenotype on a flat

substrate. (A) Representative images of rat tenocytes stained with Phalloidin-568 to visualize F- 
actin filaments (Magenta-artificial color) and counterstained with DAPI to visualize the nucleus (Cyan-artificial color) cultured on flat surfaces from passage 1 (P1) until passage 5 (P5). (B) Changes in cell area, aspect ratio, compactness and solidity from P1 to P5 (*P $<0.05$, **P < $0.01, * * * \mathrm{P}<0.005, * * * * \mathrm{P}<0.0001)$. (C) Changes in nucleus area, aspect ratio and solidity from $\mathrm{P} 1$ to $\mathrm{P} 5(* * \mathrm{P}<0.01, * * * \mathrm{P}<0.005, * * * * \mathrm{P}<0.0001)$. (D) Expression of tenogenic marker genes Scleraxis (Scx), Collagen type 1 (Collal), Collagen type three (Col3), Decorin (Dcn) and Biglycan (Bgn) from P1 towards P5 all decreased with passage number. A $\log _{10}$ transformation of expression fold changes is used to visualize the differences in gene expression. Scale bars represent $100 \mu \mathrm{m}$. (Error bars represent $95 \%$ confidence intervals, $* \mathrm{P}<0.05, * * \mathrm{P}<0.01$ ). For all experiments, $\mathrm{n}=3$. 
bioRxiv preprint doi: https://doi.org/10.1101/2020.07.23 217224: this version posted July 24,2020 . The copyright holder has placed this preprint (which was not certified by peer review) in the Public Domain. It is no longer restricted by copyright. Anyone can legally share, reuse, remix, or adapt this material for any purpose without crediting the original authors.

A

(1)

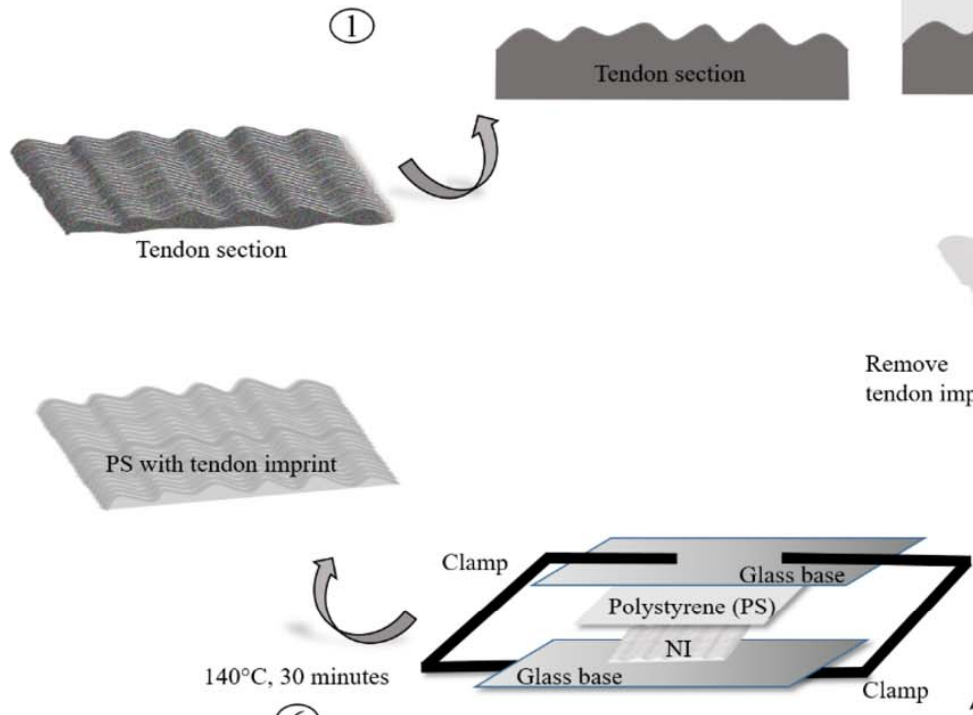

(6)
(2)

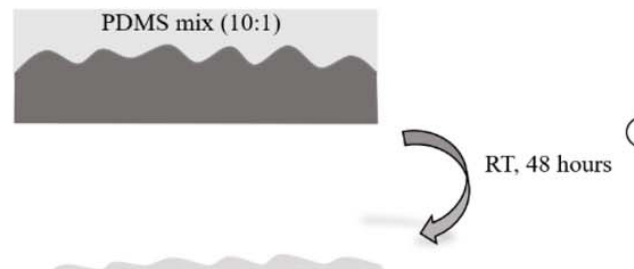

(3)

(4)

B

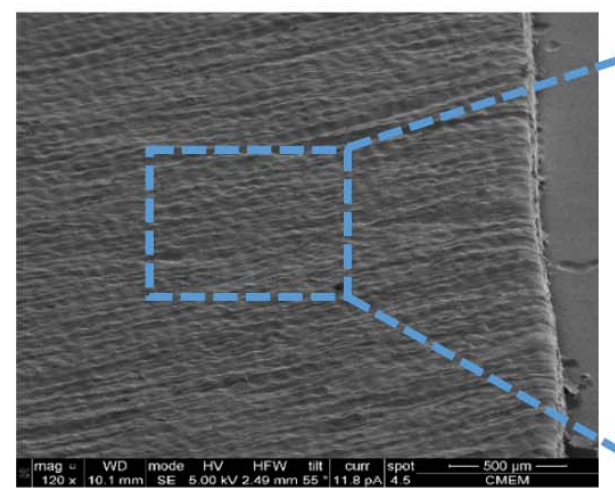

C

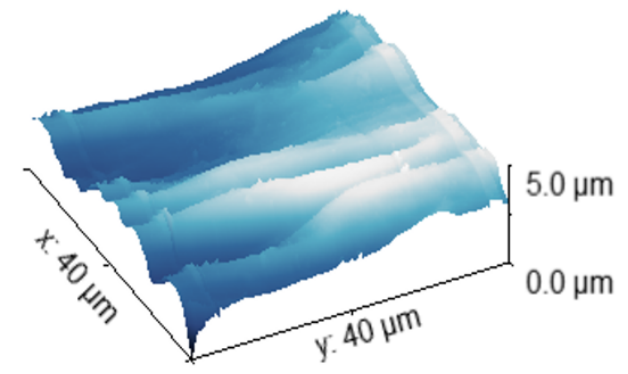

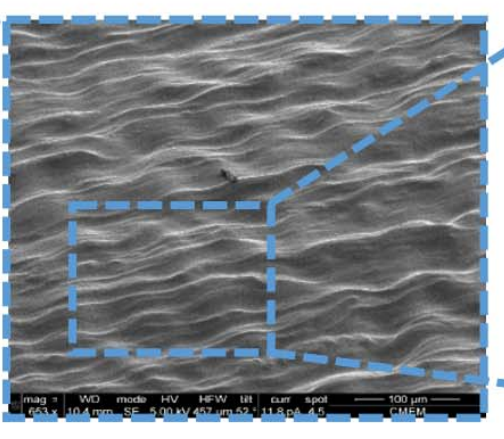

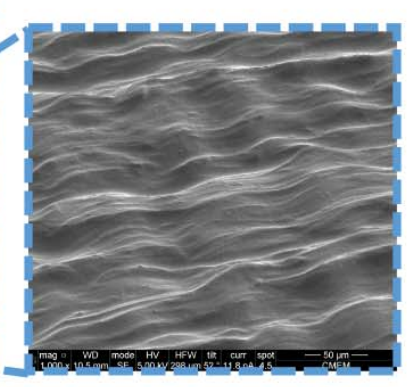

(5)

PDMS negative imprint of tendon section (NI)

Place PS on NI and clamp between two glass slides

D
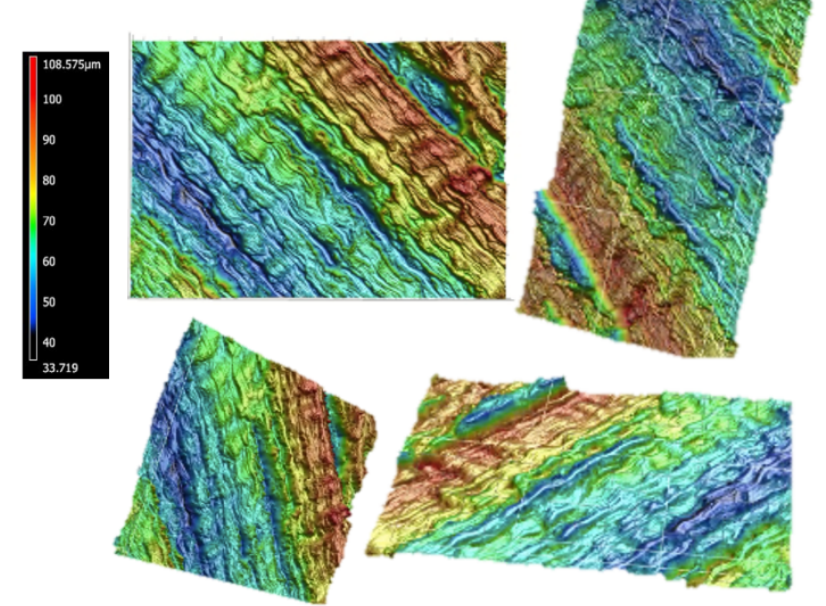
Figure 2. The native tendon imprinted as a cellular niche on polystyrene. (A) Procedure to develop a native tendon imprint, using an embossing technique of a native tendon tissue into polystyrene. (B) SEM image of tendon imprint with lower (left) and higher (right) magnification. The red dotted box represents a randomly selected region on the imprint. (C) Representative AFM image of tendon imprint showing that the imprint can reach a depth of $5 \mu \mathrm{m}$ height. (D) Representative image of profilometer images of tendon imprint, depicted from different angles. 


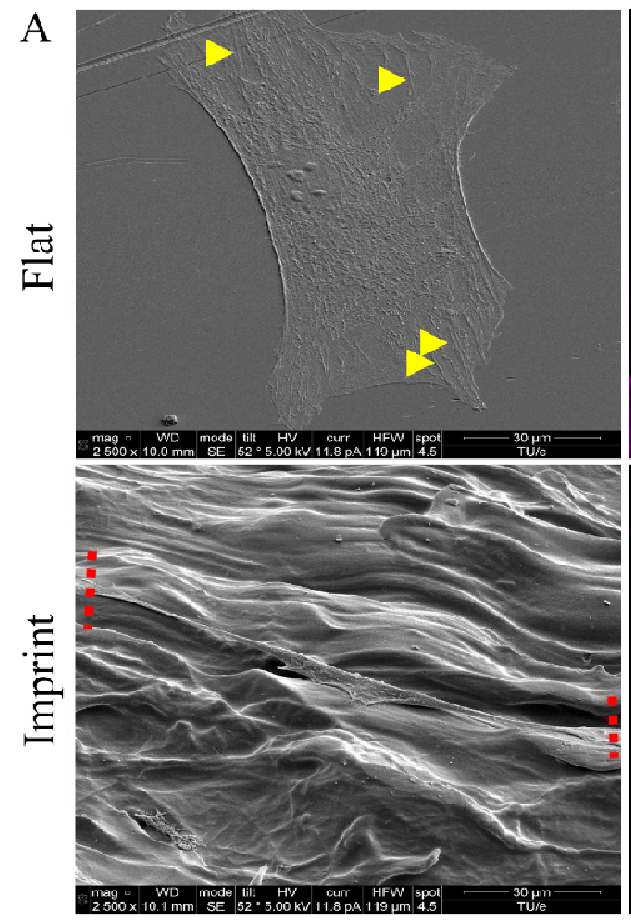

D

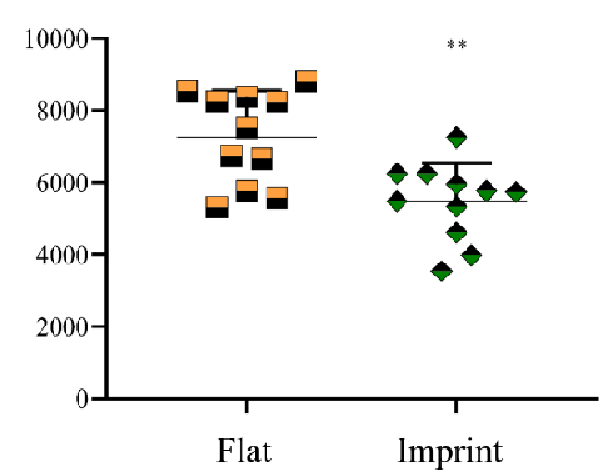

E

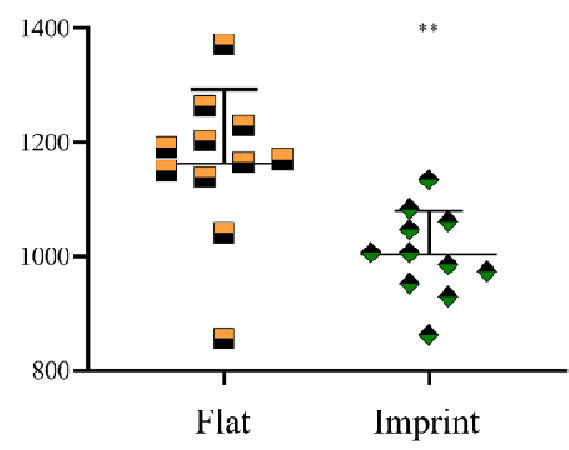

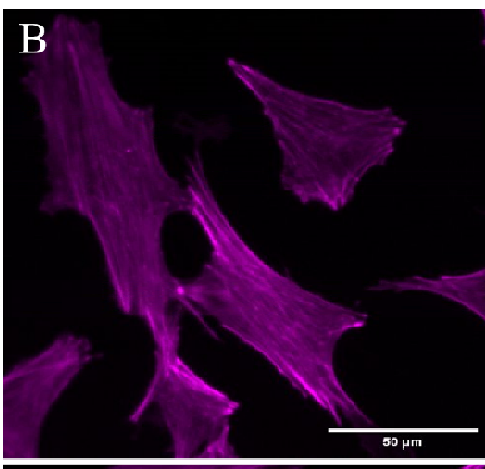
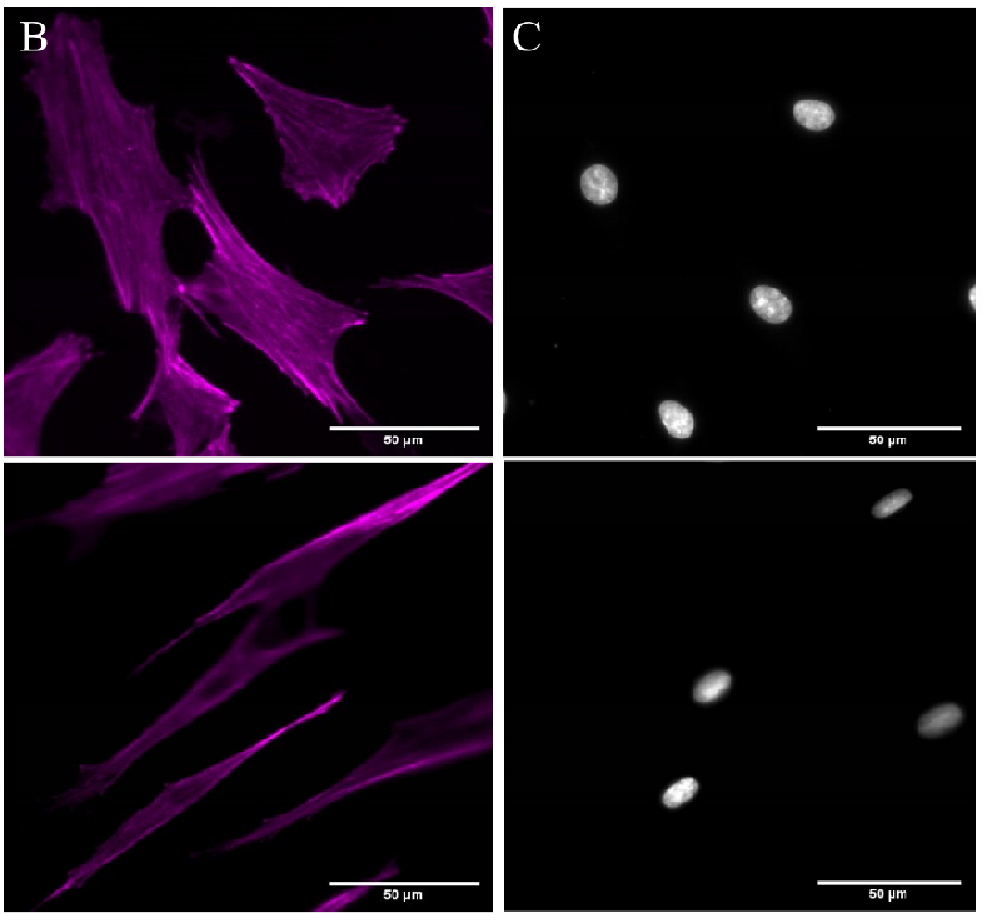

Cell shape parameters

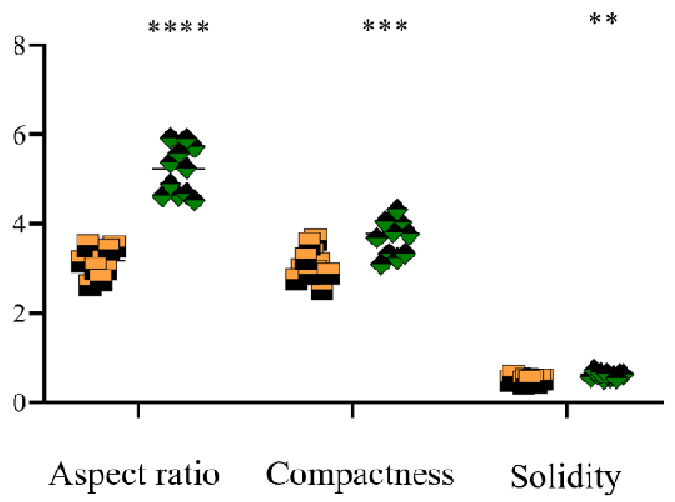

Nucleus shape parameters

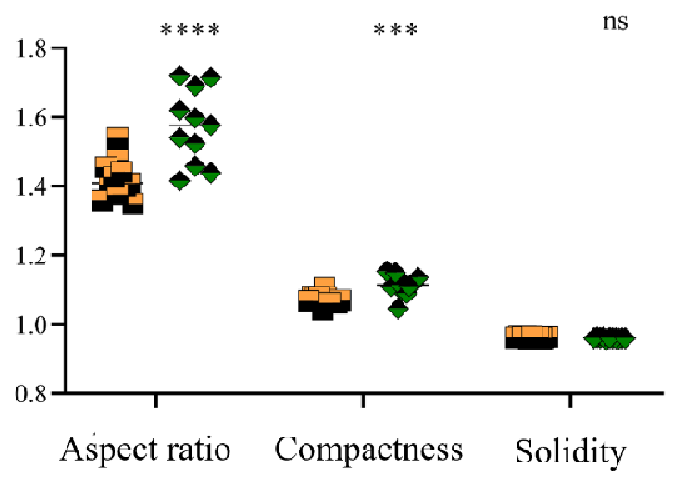

E Flat

- Imprint 


\section{Figure 3. Tendon imprint topography manipulates tenocytes to their natural morphology}

Tendon imprint topography pushes tenocytes to their natural morphology (A) Representative images of tenocytes cultured on the flat surface (left) and four replicates of the tendon of tendon imprints (right). Top images belong to SEM imaging, bottom images belong to fluorescent staining (Phalloidin in Gray, artificial color. DAPI in blue). Scale bars represent $100 \mu \mathrm{m}$. (B) SEM images of tenocytes cultured on a flat surface (top) and tendon imprint (bottom). Yellow arrows represent stress fibers on the tenocytes on the flat surface. Red dotted lines represent the major axis boundaries of tenocytes. Scale bars represent $30 \mu \mathrm{m}$. (C) Representative images of cell shape (magenta, artificial color) of rat tenocytes cultured on flat (top) and tendon imprint (bottom). Scale bars represent $10 \mu \mathrm{m}$. (D) Representative images of nucleus shape (grey, artificial color) of rat tenocytes cultured on flat (top) and tendon imprint (bottom). Scale bars represent 10 $\mu \mathrm{m}$. (E) Cell shape parameters are significantly different between both flat and imprint topographies, except for nuclear solidity $(* \mathrm{p}<0.05)$. For all experiments, $\mathrm{n}=3$.
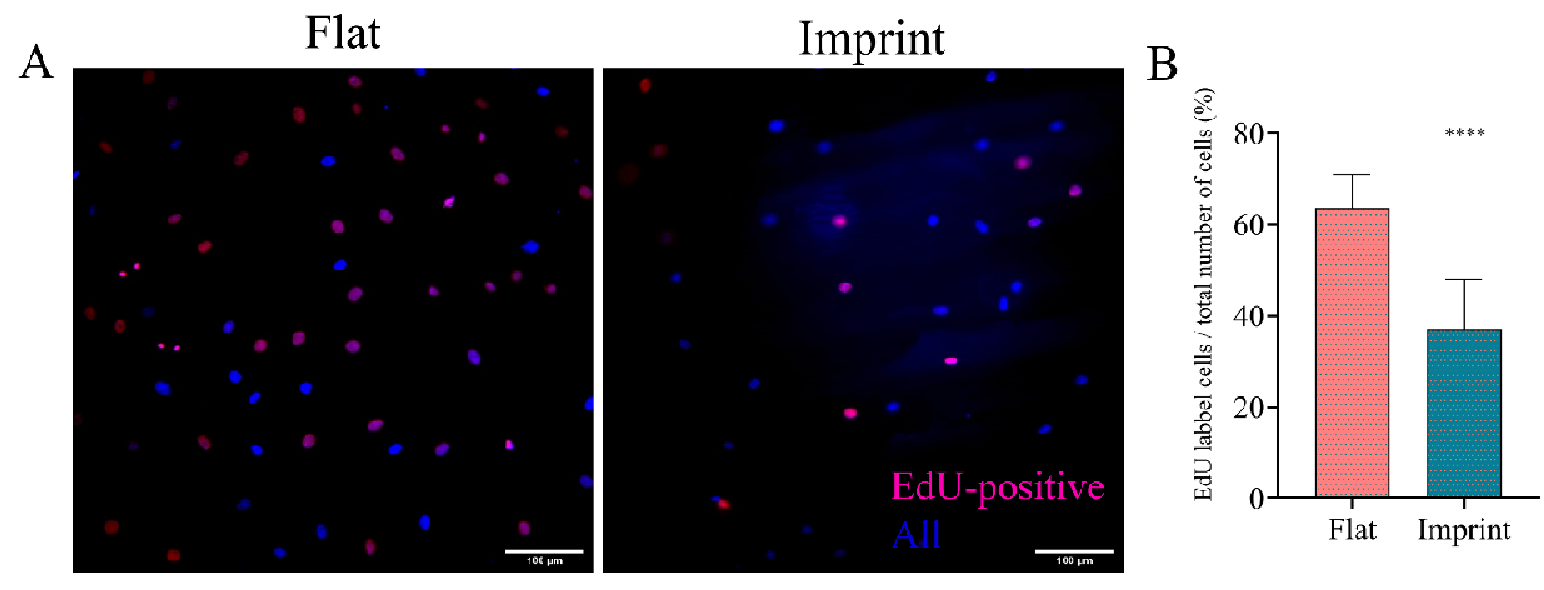

Figure 4. Tendon imprint topography lower cell proliferation capacity. (A) Representative images of EdU staining in which proliferating cells are labelled in pink-purple color and Hoechst is used as a counterstain (in blue color). (B) Quantification of EdU positive tenocytes on the flat 
bioRxiv preprint doi: https://doi.org/10.1101/2020.07.23.217224; this version posted July 24, 2020. The copyright holder has placed this preprint (which was not certified by peer review) in the Public Domain. It is no longer restricted by copyright. Anyone can legally share, reuse, remix, or adapt this material for any purpose without crediting the original authors.

surface and tendon imprints. On the flat surface, $63 \%$ of the cells was EdU positive, whereas on tendon imprints it was only 37\%. Scale bars represent $100 \mu \mathrm{m}$. (Error bars represent 95\% confidence intervals, $* \mathrm{p}<0.05)$. For all experiments, $\mathrm{n}=3$. 

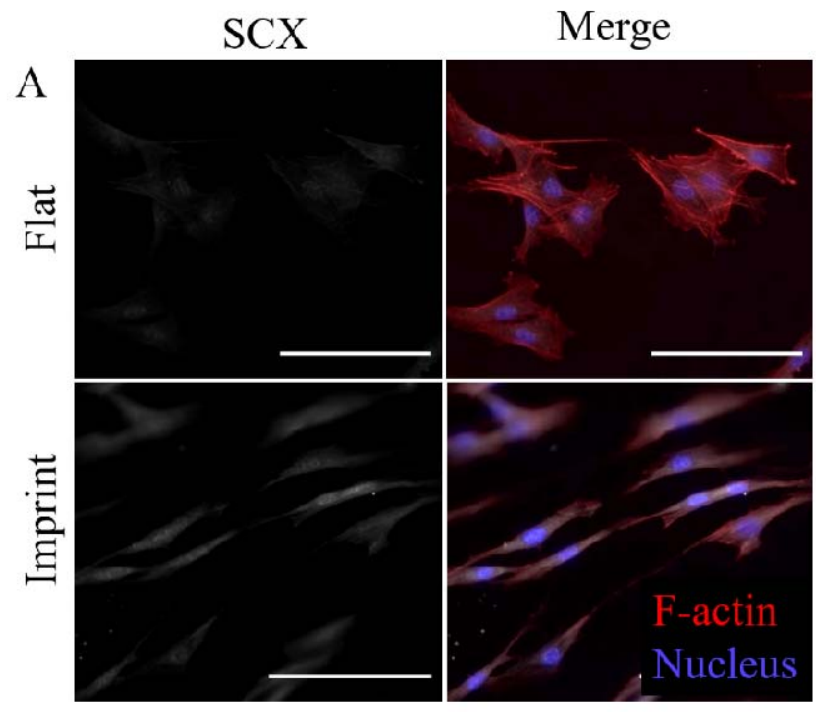

B SCX intensity (A.U)

$\mathrm{C}$
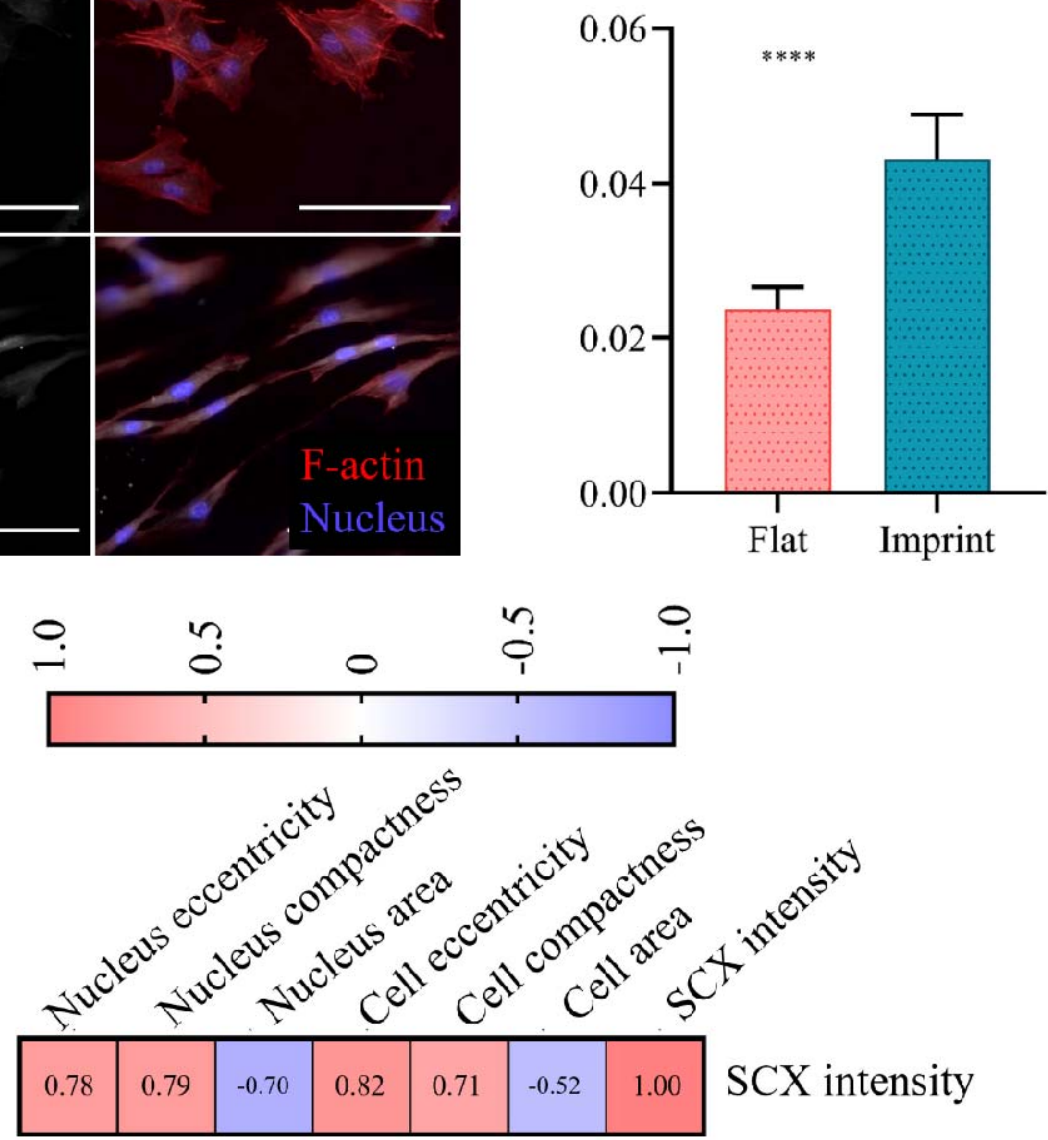

Correlation coefficient (rs)

D
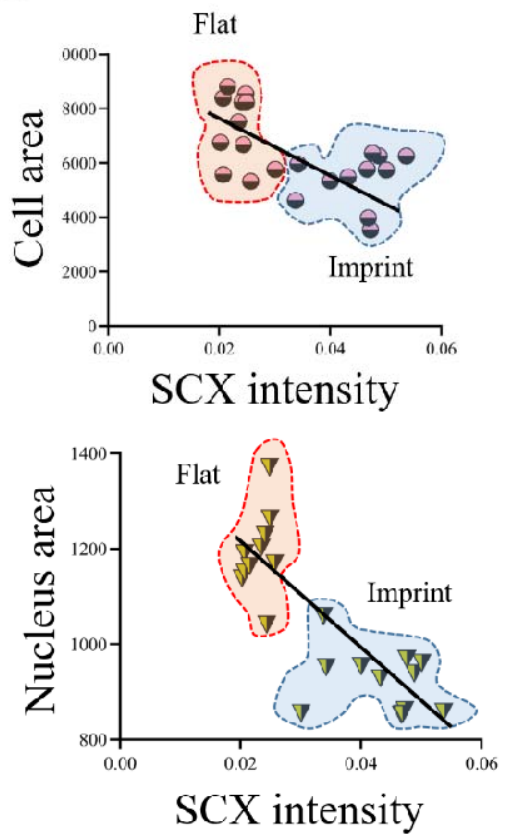
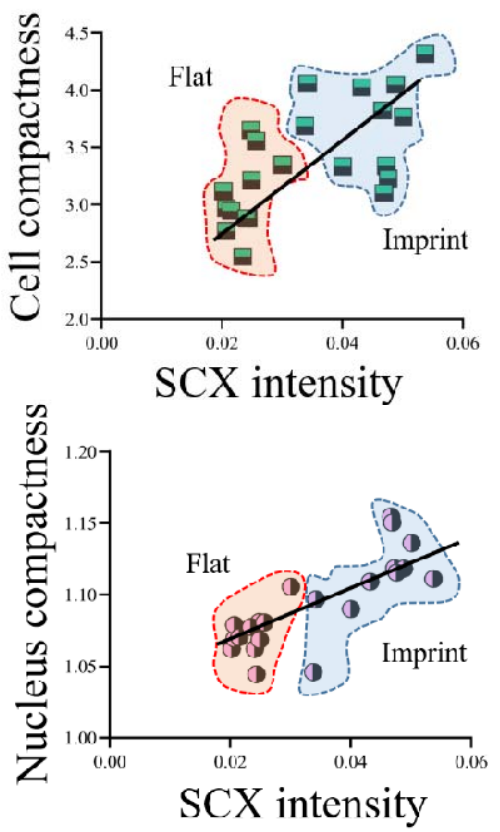
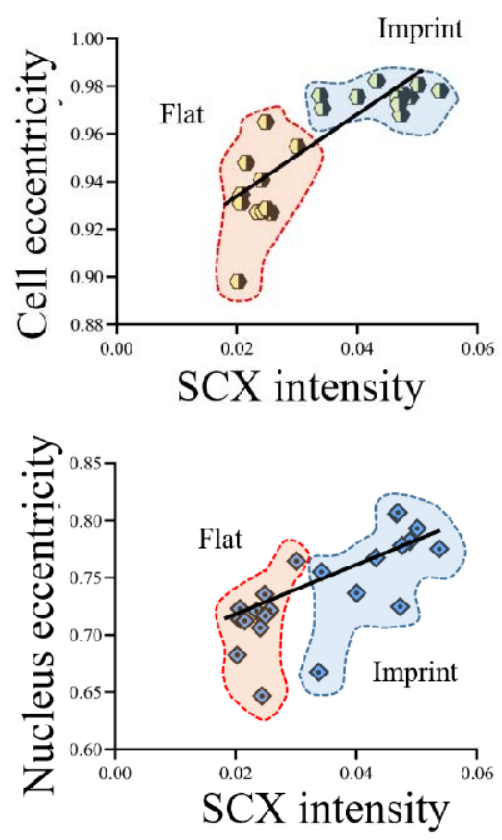


\section{Figure 5. Expression of SCX correlates with cellular and nuclear features.}

Tenocyte phenotype is maintained by tendon imprints (A) Representative images of rat tenocytes cultured for 24 hours on a flat surface and a tendon imprint and stained for SCX. (B) Quantification of SCX level in tenocytes on flat surface and tendon imprint. (C) Levels of SCX correlates with cellular and nuclear features Spearmen correlation values $\left(r_{s}\right)$ between SCX levels and eccentricity, compactness and area of cell and nucleus. (D) X-Y graphs of cell and nuclear area, compactness and eccentricity correlated with SCX level. Each data point represents the median value of individual intensities of all cells measured in a single image. Area indicated in red and blue belong to cells on flat and imprint surfaces, respectively. For visual purposes, a trend line was added derived from a simple linear regression calculation.

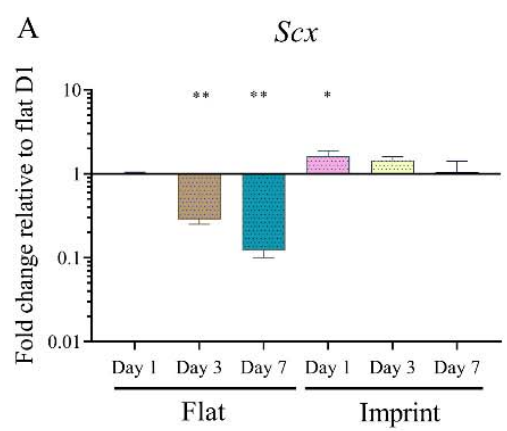

B
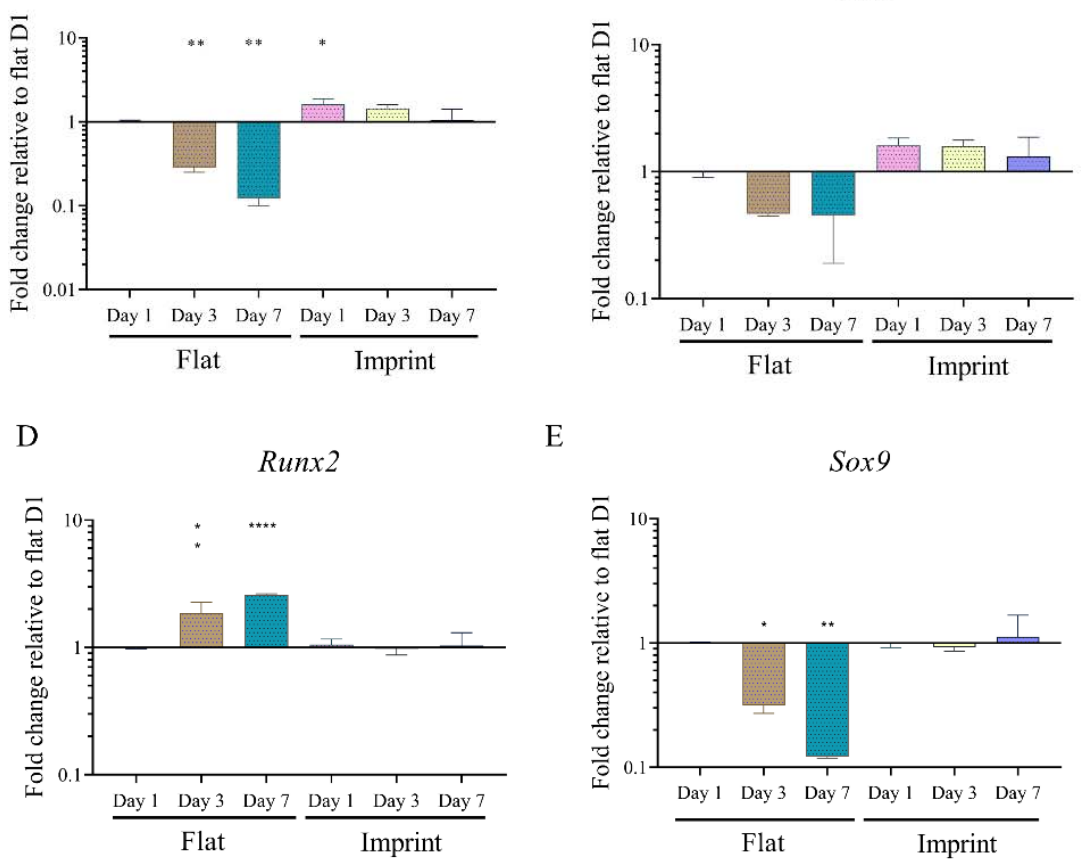

E

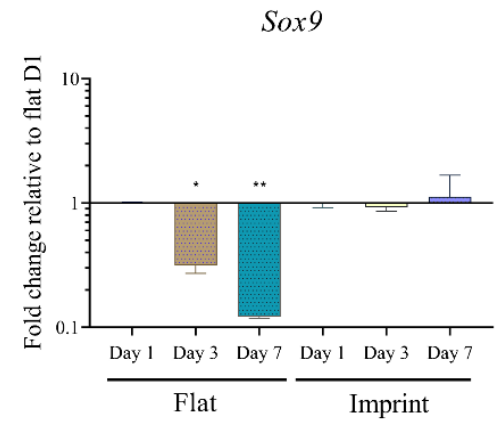

$\mathrm{C}$

Collal

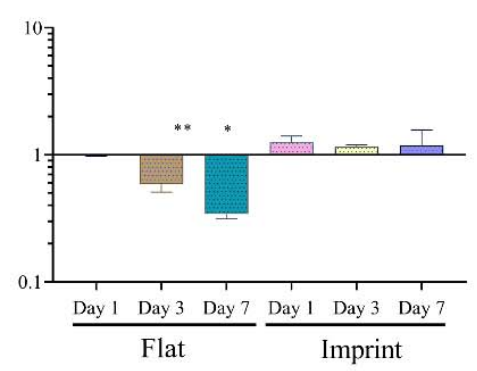

Flat Imprint

Figure 6. Tenocyte phenotype is maintained on tendon imprint topography Gene expression levels of (A) Scx, (B) Dcn, (C) Colla1, (D) Runx2 and (E) Sox9 of tenocytes cultured on flat 
bioRxiv preprint doi: https://doi.org/10.1101/2020.07.23.217224; this version posted July 24,2020 . The copyright holder has placed this preprint (which was not certified by peer review) in the Public Domain. It is no longer restricted by copyright. Anyone can legally share, reuse, remix, or adapt this material for any purpose without crediting the original authors.

surfaces and tendon imprints over 7 days of culture. On a flat surface, expression of Scx, Dcn, Colla1, Sox9 decrease whereas Runx2 increases. Contrary, tendon imprints preserved tenocyte phenotype. A $\log _{10}$ transformation of the expression fold changes is used to visualize the differences in gene expression. (Error bars represent $95 \%$ confidence intervals, $* \mathrm{P}<0.05$, $* * \mathrm{P}<$ 0.01). For all experiments, $\mathrm{n}=3$. 
bioRxiv preprint doi: https://doi.org/10.1101/2020.07.23.217224; this version posted July 24, 2020. The copyright holder has placed this preprint (which was not certified by peer review) in the Public Domain. It is no longer restricted by copyright. Anyone can legally share, reuse, remix, or adapt this material for any purpose without crediting the original authors.

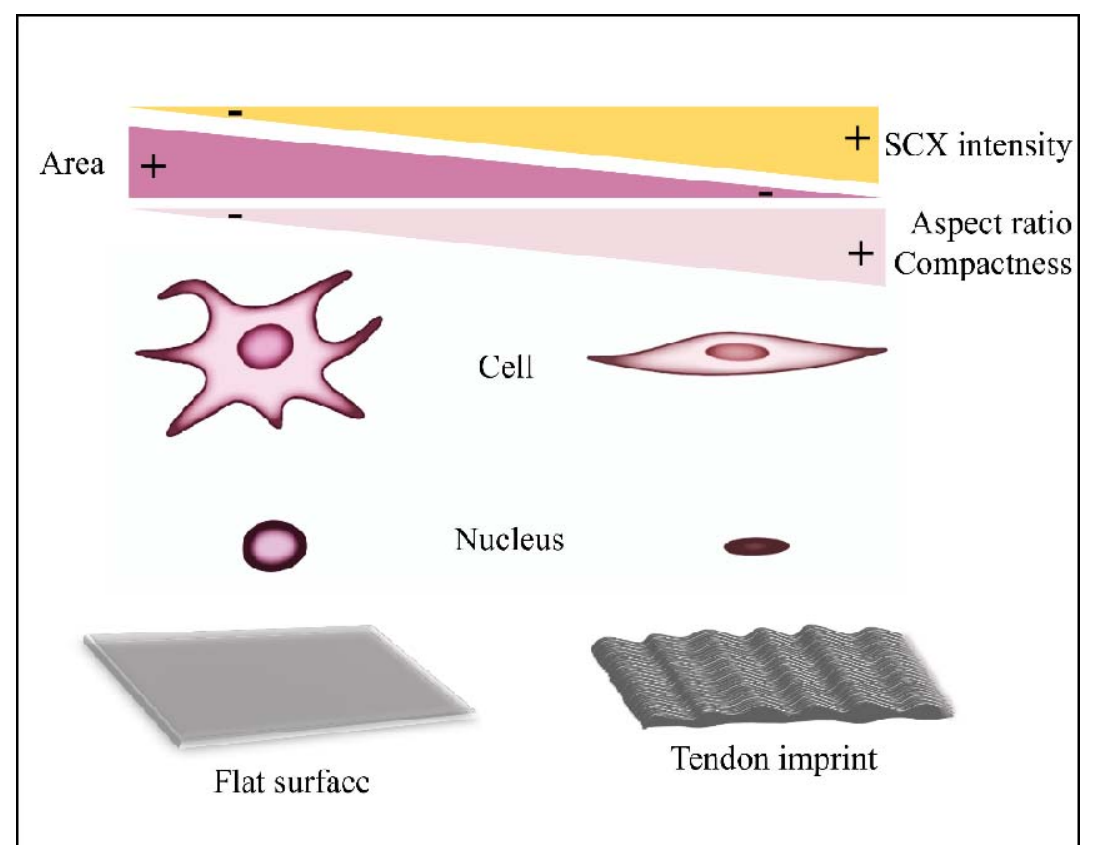

Figure 7. Summary of the results. Tenocytes on imprint surfaces display a lower cell area and higher aspect ratio, compactness and solidity, which results in increased SCX intensity. 